\title{
Prognosis of Abdominal Pain in Children in Primary Care-A Prospective Cohort Study
}

Yvonne Lisman-van Leeuwen, $P b D^{1,2}$

Leo A. A. Spee, $M D^{2}$

Marc A. Benninga, $M D, P b D^{c 3}$

Sita M. A. Bierma-Zeinstra, PbD ${ }^{2}$

Marjolein Y. Berger MD, $P b D^{1,2}$

'Department of General Practice, University of Groningen, University Medical Center Groningen, Groningen, the Netherlands

${ }^{2}$ Department of General Practice, Erasmus $\mathrm{MC}$, Rotterdam, the Netherlands

${ }^{3}$ Department of Paediatric Gastroenterology, Emma Children's hospital-Academic Medical Center, Amsterdam, the Netherlands

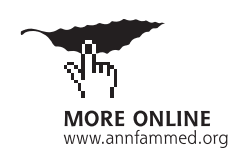

Conflict of interest: authors report none.

\section{CORRESPONDING AUTHOR}

Yvonne Lisman-van Leeuwen

Department of General Practice, FA21

University of Groningen, University

Medical Center Groningen

PO Box 196, 9700 AD

Groningen, the Netherlands

Y.Lisman-van.Leeuwen@umcg.nl

\begin{abstract}
PURPOSE Abdominal pain is a common complaint in children. Because few data exist on its natural history, we wanted to investigate the prognosis of abdominal pain in children in general practice.
\end{abstract}

METHODS In a prospective cohort study of children (aged 4 to 17 years) complaining of abdominal pain, follow-up was at 3,6, 9, and 12 months using standardized questionnaires. The primary outcome measure was chronic abdominal pain: abdominal pain at least 1 time a month during at least 3 consecutive months that had an impact on daily activities. Prevalence, incidence, and duration of chronic abdominal pain were assessed.

RESULTS Three hundred five children (116 boys, 189 girls), with a median age of 7.8 years (interquartile range $[\mathrm{IQR}]=5.7-10.5$ years) were included. Chronic abdominal pain was present in $142(46.6 \%)$ children at an initial visit to the primary care physician for this complaint. During follow-up, $78.7 \%$ fulfilled the criteria for chronic abdominal pain at 1 or more follow-up points. Among 163 children at risk for developing chronic abdominal pain, the cumulative incidence of chronic abdominal pain was $60.1 \%$ (95\% Cl, 52.1\%-67.7\%) and was higher in girls than in boys ( $R R=1.23 ; 95 \% \mathrm{Cl}, 0.94-1.61)$. Median duration of abdominal pain was 7.5 months (IQR $=4.5-12.0$ months). Children aged 10 to 17 years had the longest duration of abdominal pain (median $=9.0$ months; IQR $=7.5$ 12.4 months). Children with symptoms of irritable bowel syndrome seemed to have a less favorable prognosis compared with children with symptoms of functional dyspepsia or functional abdominal pain.

CONCLUSIONS The presence and development of chronic abdominal pain is common and of long duration among children consulting in primary care for abdominal pain. These poor outcome data warrant follow-up.

Ann Fam Med 2013;11:238-244. doi:10.1370/afm.1490.

\section{INTRODUCTION}

$\mathrm{P}$ rimary care physicians are frequently consulted for abdominal pain in children. ${ }^{1,2}$ In some children abdominal pain becomes a chronic problem. The prevalence of chronic abdominal pain (CAP) in samples of school-based children in Western countries ranges from $0.3 \%$ to $19 \% .{ }^{3}$ Usually the pain is functional, meaning that no objective evidence for an underlying organic disorder can be found. ${ }^{4,5}$ CAP has major implications for the child's well-being and use of the health care system. ${ }^{6,7}$

In their landmark study, Apley and Naish defined CAP as 3 bouts of abdominal pain severe enough to affect daily activities and occurring over a period of not less than 3 months. ${ }^{4}$ Because of ambiguities in this definition, Von Baeyer and Walker proposed a new, more precise definition, which was a precursor for the pediatric Rome III diagnostic criteria. ${ }^{8,9}$ According to their definition, a child suffers from CAP if abdominal pain appears at least once a month in 3 consecutive months, and the pain has an impact on the child's daily activities or well-being. ${ }^{8}$ According to the Rome III criteria, functional CAP is recognized as an abdomi- 
nal pain-related functional gastrointestinal disorder (FGID), which can be subdivided into functional dyspepsia, irritable bowel syndrome, abdominal migraine, and functional abdominal pain. ${ }^{9}$ Functional abdominal pain syndrome refers to children with functional abdominal pain and additional somatic symptoms that affect daily activities.

There are few data on the natural history of abdominal pain in children, and only a few studies have followed up on children with CAP into adolescence and adult life. A systematic review showed that in 29.1\% of children with CAP, abdominal complaints persisted after a median follow-up of 5 years. ${ }^{10}$ Most studies of CAP are performed in secondary or tertiary care, however, and these referred children likely represent a selected population of children with more severe pain.

To date there is a lack of data on the occurrence of CAP in children complaining of abdominal pain in primary care. Information on the course of abdominal pain and on factors influencing the course is essential to establish which child needs further treatment and which child will improve over time with no treatment other than reassurance. ${ }^{11-13}$ This study describes the prognosis of abdominal pain in children in primary care.

\section{METHODS}

\section{Study Design and Patients}

We undertook a prospective cohort study with 12 months of follow-up. Children were recruited consecutively between May 2004 and March 2006 by 53 primary care physicians in Rotterdam and surroundings. ${ }^{14}$ Children aged 4 to 17 years could participate if they visited their primary care physician with the complaint of abdominal pain. Children who had consulted their physician for abdominal pain in the preceding 3 months, children who had a diagnosis of inflammatory bowel disease, celiac disease, or lactose intolerance before baseline, and children unable to complete questionnaires because of language or cognitive problems were excluded. Within 1 week after their consultation, a research assistant visited the children and obtained informed consent from the child and parent(s). At this visit several questionnaires were completed by the parents and the child, if the child was aged 9 years to 17 years, addressing sociodemographic characteristics, presence and duration of symptoms, and medical and family history. The questionnaires are described in detail elsewhere. ${ }^{14}$

Follow-up was at 3, 6, 9, and 12 months. All questionnaires used at baseline were completed again at 12 months, whereas at 3,6, and 9 months only the part of the questionnaire asking about the presence and duration of symptoms was filled out. All follow- up questionnaires were sent by regular mail. At 3 months, the physicians' final diagnosis was extracted from the physician-maintained electronic medical records. The Central Committee on Research Involving Human Subjects in the Netherlands approved the study. We evaluated possible selective recruitment and observed that this cohort represented young schoolaged children consulting their primary care physician for a new episode of abdominal pain not diagnosed as gastroenteritis. $^{14}$

\section{Analyses}

To investigate prognosis, we determined how often abdominal pain did not resolve and turned into CAP. We calculated prevalence, incidence, duration, and number of recurrences of CAP among the children included in our cohort, ie, children complaining of abdominal pain in general practice. CAP was defined by the criteria of Von Baeyer and Walker as having abdominal pain at least once a month during at least 3 consecutive months. ${ }^{8}$ In addition, abdominal pain had to interfere with daily activities, which was defined as abdominal pain severe enough to stay home from school, to terminate or avoid play (absence), to take medication (medication), or rating the pain intensity as moderate to severe (pain intensity). Pain intensity was determined with a numerical rating scale in children aged 8 to 17 years ranging from 0 (no pain) to 10 (worst pain possible). In children aged 4 to 8 years, we used the pain faces scale: 6 faces ranging from happy or no pain at all (0) to the worst pain you can imagine (10). ${ }^{15}$ Moderate to severe abdominal pain was defined as a score of 3 or higher for children aged 8 to 17 years and 4 or higher for children aged 4 to 8 years. ${ }^{8}$

The prevalence of CAP was determined at baseline and all follow-up points. The prevalence of both components of CAP (abdominal pain and impact) was determined separately. The incidence of CAP was determined during follow-up among children without CAP at baseline. The duration of CAP during followup was calculated as the total number of consecutive months that the child had CAP. If data were missing at 1 follow-up point, we extrapolated the duration between the follow-up points before and after the missing point except when it concerned missing data at 12 months. In the latter case the child was considered lost to follow-up. A maximum duration of 15 months could be achieved, because we elicited information about abdominal pain in the 3 months preceding baseline and at each follow-up point.

For children with CAP at baseline, we calculated the duration of CAP and the number of recurrences. We then determined the influence of age, sex, diagnosis, and presence of symptoms resembling FGIDs 
at baseline on prevalence, incidence, and duration of CAP. Age was categorized into groups: 4 to 5 years, 6 to 9 years and 10 to 17 years. ${ }^{16}$ We categorized children on the basis of their symptoms at baseline as resembling the symptoms of functional dyspepsia, irritable bowel syndrome, or functional abdominal pain. Symptoms of these FGIDs were defined by the Rome III criteria irrespective of the duration of symptoms

(Supplemental Appendix 1, available at http:// annfammed.org/content/11/3/238/suppl/DC1). ${ }^{9}$

\section{Statistical Analyses}

We used descriptive statistics and report prevalence, cumulative incidence, and cumulative incidence ratio (CIR) with their 95\% confidence intervals. Prevalence was calculated as the number of children with CAP divided by the total number of children at that followup time. Cumulative incidence was calculated by dividing the number of children who developed CAP during follow-up by the number of children without CAP at baseline. The cumulative incidence reflects the risk. The CIR is the ratio of 2 cumulative incidences and reflects the risk ratio. The duration of CAP is displayed as median duration with interquartile range (IQR). Differences in medians were tested with the MannWhitney test or Kruskall Wallis test, differences in proportions with the $\chi^{2}$ test. Analyses were performed using SPSS Predictive Analytics SoftWare (PASW) (SPSS 18.0, SPSS Inc).

\section{RESULTS}

A total of 348 children consented to be approached for the study. Of these, 305 (87.6\%) children participated in the study: their median age was 7.8 years $(I Q R=5.7-$ 10.5 years); 116 were boys $(38.0 \%)$ and 189 were girls (62.0\%). Table 1 shows the baseline characteristics, and Figure 1 displays the patient flow and reasons for nonparticipation. The 22 children lost to follow-up did not differ from the children included with respect to age, sex, and the presence of CAP at baseline.

\section{Prevalence of CAP Among Children With Abdominal Pain}

A total of 142 children $(46.6 \%)$ had CAP at the first visit, compared with 105 children $(37.1 \%)$ at the 12-month follow-up (Figure 2). In total, 240 of 305 children $(78.7 \%)$ fulfilled the criteria for CAP at 1 or more follow-up periods. At each follow-up period, the prevalence of CAP was higher in girls than in boys, although only at 3 and 6 months was this statistically significant $(P=.008$ and $P=.006$, respectively). Compared with baseline the prevalence at 12 months was decreased in children aged 4 to 5 years and 6 to
9 years $(P=.034$ and $P=.01$, respectively), but not in children aged 10 to 17 years $(P=.325)$ (Table 2$)$. The prevalence of CAP according to the FGID symptom classification is shown in Table 3. During follow-up the prevalence of CAP decreased significantly for children with dyspepsia or functional abdominal pain symptoms ( $P=.029$ and $P<.001$, respectively) but not for children with symptoms of irritable bowel syndrome $(P=.134)$.

Most children fulfilled the impact criteria because of moderate to severe pain (Table 4). The percentage of boys and girls for whom their symptoms had an impact at baseline was similar $(P=.867)$. Although boys were impact-free earlier, there was no difference in impact between boys and girls after 12 months $(P=.349)$. The highest prevalence of impact at all follow-up periods was seen in children aged 10 to 17 years. After 12 months $61.0 \%$ of children aged 10 to 17 years fulfilled the impact criteria vs $46.3 \%$ and $43.8 \%$ in children aged 6 to 9 years and 4 to 5 years, respectively $(P=.028)$. There was no association between impact and FGID symptom classification.

\section{Table 1. Baseline Characteristics of the $\mathbf{3 0 5}$} Participating Children

\begin{tabular}{|c|c|}
\hline Characteristic & Baseline \\
\hline Age, median (IQR), years & $7.8(5.7-10.5)$ \\
\hline \multicolumn{2}{|l|}{ Age category, No. (\%) } \\
\hline 4-5 years & $88(28.9)$ \\
\hline $6-9$ years & $126(41.3)$ \\
\hline$\geq 10$ years & $91(29.8)$ \\
\hline \multicolumn{2}{|l|}{ Sex, No. (\%) } \\
\hline Boys & $116(38.0)$ \\
\hline Girls & $189(62.0)$ \\
\hline \multicolumn{2}{|l|}{ Diagnosis of physician, No. (\%) } \\
\hline Generalized abdominal pain & $174(57.0)$ \\
\hline Localized abdominal pain & $57(18.7)$ \\
\hline Constipation & $31(10.2)$ \\
\hline Gastroenteritis & $17(5.6)$ \\
\hline Urinary tract infection/cystitis & $5(1.6)$ \\
\hline Stomach pain & $2(0.7)$ \\
\hline Appendicitis & $1(0.3)$ \\
\hline Other $^{\mathrm{a}}$ & $10(3.3)$ \\
\hline No diagnosis available & $8(2.6)$ \\
\hline \multicolumn{2}{|l|}{ Symptom classification, No. (\%) } \\
\hline Functional dyspepsia & $24(7.9)$ \\
\hline Irritable bowel syndrome & $114(37.4)$ \\
\hline Functional abdominal pain & $152(49.8)$ \\
\hline Missing classification & $15(4.9)$ \\
\hline \multicolumn{2}{|c|}{ ICPC = International Classification of Primary Care. } \\
\hline \multicolumn{2}{|c|}{$\begin{array}{l}\text { a Other diagnoses were acute upper respiratory tract infection (ICPC code R74, } \\
n=3) \text {, otitis media }(H 71, n=1) \text {, hernia inguinalis sinistra }(D 89, n=1) \text {, pneu- } \\
\text { monia (R81, } n=1) \text {, mononucleosis infection (A75, } n=1) \text {, other viral disease } \\
\text { with exanthema }(A 76, n=1) \text {, lymphadenitis mesenterica }(n=1) \text {, fatigue/ } \\
\text { malaise }(A 04, n=1) \text {. }\end{array}$} \\
\hline
\end{tabular}


Incidence of CAP Among Children With Abdominal Pain

Of the 163 children without CAP at baseline, 98 had CAP at some point during follow-up, yielding a cumulative incidence of $60.1 \%$ (95\% CI, 52.1-67.7). The cumulative incidence in girls was $65.3 \%$ and in boys

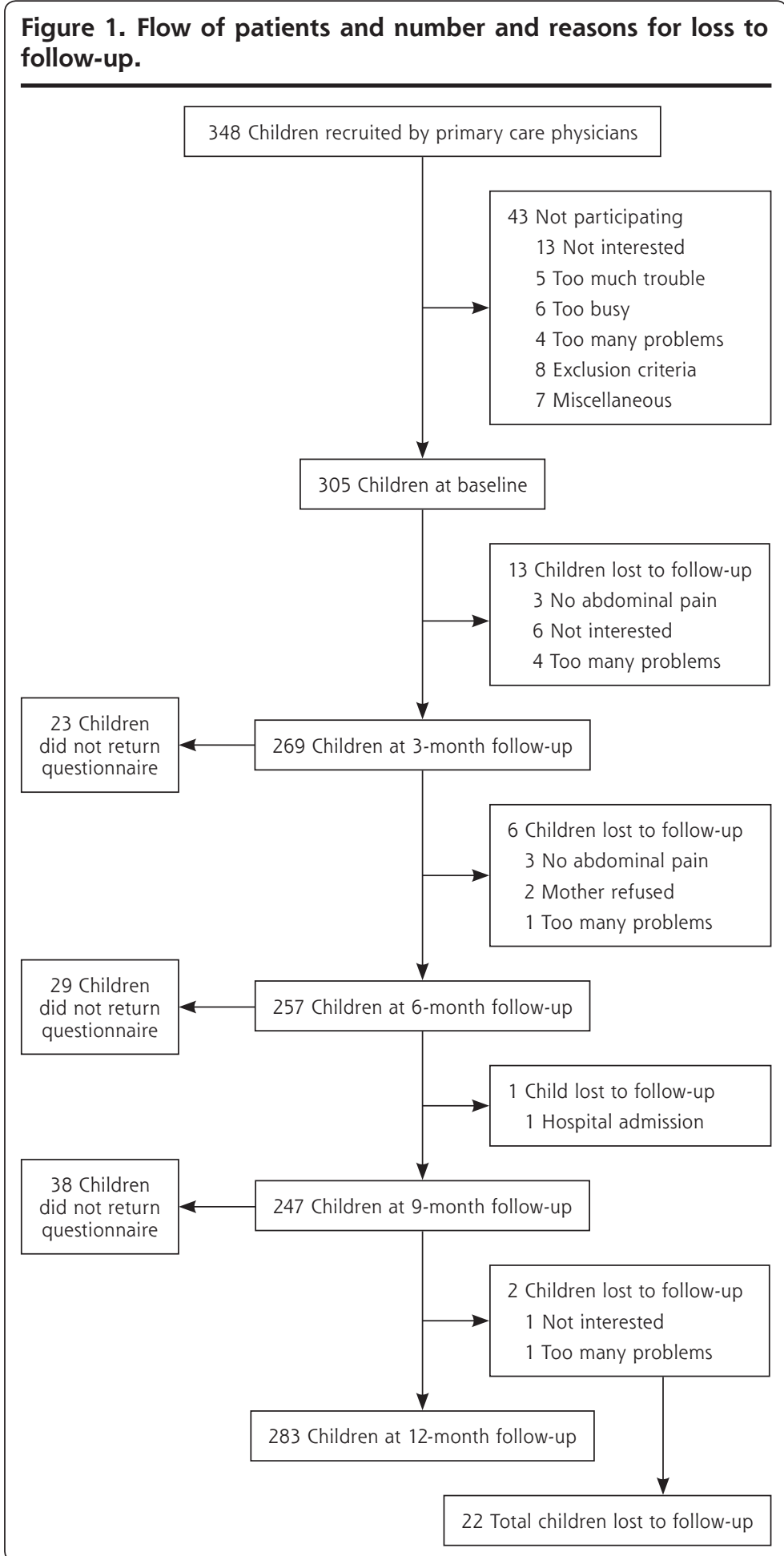

$52.9 \%$, yielding a CIR of 1.23 (95\% CI, 0.94-1.61).

Compared with children aged 4 to 5 years, the cumulative incidence in children aged 6 to 9 years was not increased $(57.8 \%$ vs $55.4 \%, \mathrm{CIR}=0.96,95 \% \mathrm{CI}, 0.67$ $1.34)$ as was for children aged 10 to 17 years $(67.9 \%$, $\mathrm{CIR}=1.18 ; 95 \% \mathrm{CI}, 0.86-1.60)$. The cumulative incidence of CAP was highest in children with functional abdominal pain (64.9\%) compared with functional dyspepsia (46.2\%, CIR $=1.54 ; 95 \% \mathrm{CI}, 0.85-2.76)$ and irritable bowel syndrome $(56.1 \%$, $\mathrm{CIR}=1.25 ; 95 \% \mathrm{CI}, 0.83-1.88)$, although not statistically significant.

\section{Duration of CAP Among Children With Abdominal Pain}

Median duration of CAP during follow-

up was 8.3 months $(\mathrm{IQR}=4.5-12.0$

months). Girls had a longer duration than boys, median 9.0 months $(\mathrm{IQR}=5.3$ 13.50 months) vs 7.5 months $(\mathrm{IQR}=1.5$ 12.0 months; $P=.002$ ). Median duration in children aged 4 to 5 years was 7.5 months (IQR $=4.5-10.5$ months) and in children aged 6 to 9 years was 7.5 months (IQR $=4.5-12.0$ months); the longest median duration was seen in children aged 10 to 17 years (9.0 months, $\mathrm{IQR}=7.5$-12.4 months). There were no significant differences in duration among the 3 age-groups $(P=.094)$. A median duration of CAP of 9.0 months (IQR $=4.5-12.0$ months) was seen in children with irritable bowel syndrome symptoms, compared with 7.5 months in children with dyspepsia or functional abdominal pain symptoms $(\mathrm{IQR}=2.3$ 12.0 months and 4.5-12.0 months, respectively) $(P=.692)$.

Median duration of CAP in children who already had CAP at baseline was 7.5 months (IQR $=4.5-13.5$ months). Of all children, 31 (24.8\%) had CAP during the total 15 months. Median duration did not differ for girls and boys $(P=.2873)$. Duration increased with increasing age ${ }_{i}$ median of 7.5 months for children aged 4 to 5 years and 6 to 9 years, and 10.5 months for children aged 10 to 17 years $(P=.051)$. FGID symptom classification was not associated with duration of CAP in children with CAP at baseline (functional dyspepsia, functional abdominal pain, 7.5 months, IQR $=4.5-13.5$; irri- 
table bowel syndrome, 7.5 months, $\left.\mathrm{IQR}=4.5-15.0_{i} P=.901\right)$. In 94 children CAP disappeared, but a recurrence was observed in 35 (37.2\%). Recurrence was higher in girls compared with boys $(40.3 \%$ vs $31.3 \%)$, but not statistically different $(P=.389)$. Recurrence was not associated with age $(P=.417)$, although compared with children aged 4 to 5 years and 6 to 9 years $(39.4 \%$ and $40.5 \%$, respectively), recurrence seemed lower in children aged 10 to 17 years $(26.3 \%)$. In children with irritable bowel syndrome, symptoms frequently recurred: $54.8 \%$. Recurrence in children with functional dyspepsia or functional abdominal pain symptoms was $22.2 \%$ and $33.3 \%$, respectively. The strata of specific symptom categories included very few patients, thereby precluding meaningful statistical analysis.

\section{DISCUSSION}

In this prospective study among 305 children complaining of abdominal pain in primary care, we found that most children develop CAP for a median of 8.3 months. Girls had CAP more frequently during follow-up than boys, and CAP duration was longer in girls. The prevalence decreased during follow-up, except for children aged 10 to 17 years. Children with symptoms of irritable bowel syndrome seemed to have a less favorable prognosis compared with children with symptoms of other FGIDs with respect to prevalence, duration, and recurrence of CAP.

To our knowledge, no other prospective study has investigated the prevalence or incidence of CAP in children with abdominal pain in primary care. Most studies have focused on children who already had CAP and investigated the prognosis and risk factors for a poor prognosis. In addition, previous studies were almost exclusively performed in secondary or tertiary care, a selected group not comparable to a primary care population. A retrospective study in an Australian general practice among children in the waiting room reported that $44 \%$ of the children experienced abdominal pain in the preceding 12 months, of whom $12 \%$ experienced abdominal pain at least once each month. ${ }^{17}$ Our research group recently showed that primary care physicians are routinely confronted with children with abdominal pain. ${ }^{2}$ Thus, data on the prevalence and incidence of CAP among children visiting primary care with abdominal pain are valuable.

In the present study we observed a higher prevalence and incidence of CAP among girls with abdominal pain compared with boys with abdominal pain. This finding is also reported by others ${ }^{4,18,19}$ but not by all groups. ${ }^{20}$ Our finding that duration of CAP was longer in girls than in boys is new. Although there was no obvious peak in incidence or prevalence in a specific age-group, we observed a longer duration of CAP with increasing age. In general, a peak prevalence of CAP is reported in children aged 4 to 6 and 9 to 11 years., ${ }^{4,19,21}$ ${ }^{23}$ One explanation for the absence of a peak prevalence in these age-groups in our study could be that there is a peak in abdominal pain in general but not specifically in CAP. The result would be an equal number of patients with CAP among children with abdominal pain, but 


\section{Table 3. Prevalence of Chronic Abdominal Pain Among 305 Children With Abdominal Pain in General Practice, by Classification of Symptoms Resembling Functional Gastrointestinal Disorders}

\begin{tabular}{lrrrrr}
\hline & \multicolumn{5}{c}{ Children With Chronic Abdominal Pain, No. (\%) } \\
\cline { 2 - 6 } Symptom Category & Baseline & 3 Months & 6 Months & 9 Months & 12 Months \\
\hline Functional dyspepsia & $11(45.8)$ & $12(54.5)$ & $9(45.0)$ & $6(28.6)$ & $6(26.1)$ \\
Irritable bowel syndrome & $48(42.1)$ & $54(54.0)$ & $46(46.5)$ & $50(52.6)$ & $45(41.3)$ \\
Functional abdominal pain & $75(49.3)$ & $77(58.8)$ & $60(48.8)$ & $51(44.0)$ & $51(37.2)$ \\
Missing & $8(53.3)$ & $8(61.5)$ & $6(42.9)$ & $6(42.9)$ & $3(21.4)$ \\
\hline
\end{tabular}

Table 4. The 3 Components of Impact at Each Follow-up Point

\begin{tabular}{lccccc}
\hline Component & $\begin{array}{c}\text { Baseline } \\
\text { No. (\%) }\end{array}$ & $\begin{array}{c}\text { 3 Months } \\
\text { No. (\%) }\end{array}$ & $\begin{array}{c}\text { 6 Months } \\
\text { No. (\%) }\end{array}$ & $\begin{array}{c}\text { 9 Months } \\
\text { No. (\%) }\end{array}$ & $\begin{array}{c}\text { 12 Months } \\
\text { No. (\%) }\end{array}$ \\
\hline No impact & $22(7.2)$ & $93(33.8)$ & $105(40.6)$ & $110(43.3)$ & $142(50.2)$ \\
Impact $^{a}$ & $283(92.8)$ & $176(66.2)$ & $152(59.4)$ & $137(56.9)$ & $141(49.8)$ \\
Pain intensity & $277(97.9)$ & $152(86.4)$ & $135(88.8)$ & $124(90.5)$ & $120(85.1)$ \\
Absence & $137(48.4)$ & $55(31.3)$ & $40(26.3)$ & $36(26.3)$ & $51(36.2)$ \\
Medication & $81(28.6)$ & $38(21.6)$ & $29(19.1)$ & $31(22.6)$ & $34(24.1)$ \\
\hline
\end{tabular}

a Pain intensity = rating the pain as moderate to severe; absence = staying home from school or terminating or avoiding to play; medication $=$ taking medication for the pain. we also evaluated symptoms resembling functional dyspepsia, irritable bowel syndrome, or functional abdominal pain using the Rome III criteria and their relation to prognosis. The prognosis of children with symptoms of irritable bowel syndrome was somewhat worse than for children with symptoms of the other FGIDs, as we observed no decrease in prevalence of CAP, a longer duration of CAP, and a higher percentage of recurrence. Classifying children in the different FGIDs according to their symptoms might be valuable to predict prognosis, but further research is needed.

Some selective loss to followup may have occurred, as several children were lost to follow-up because they no longer had there would be a peak when investigating the prevalence of CAP in the general population.

In our group of children aged 10 to 17 years, the prevalence of CAP did not decrease at the 12-month follow-up, and the duration of CAP was longer. On the other hand, recurrences among these children were fewer compared with younger children. Perhaps in younger children CAP is more often recurrent, whereas in older children CAP is more often chronic.

When the children first consulted their primary care physician (baseline), almost all children fulfilled the criteria for impact of abdominal pain, suggesting that the impact on the children might be an important reason to consult their physician. With time the percentage of patients with abdominal pain decreased, but at 12 months after the initial consultation, 50\% still reported abdominal pain had an impact on daily activities. This percentage was as high as $61.7 \%$ in children aged 10 to 17 years, which indicates that in children of this age-group, not only did the abdominal pain tend to be more chronic, but also that it tended to give more trouble in daily life.

We used the definition of CAP by Von Baeyer and Walker for our study. ${ }^{8}$ According to the Rome III criteria, a child has functional abdominal pain syndrome if organic disease has also been ruled out; however, we did not rule out this criterion, because we were interested in abdominal pain irrespective of its cause. Nevertheless, only few children had organic disease according to the diagnosis of their physician. In addition to using the definition by Von Baeyer and Walker, abdominal pain. Consequently, the reported prevalence and incidence of CAP in each group might be overestimated. Because the numbers lost to follow-up were few, we believe that any possible overestimations are clinically irrelevant. Another limitation is that we calculated the duration of CAP during 1 year after the initial consultation to the primary care physician. We do not have information on how long the child experienced abdominal pain before entry into the study or after follow-up ended, so the exact duration therefore remains unknown. For the physician, however, it is valuable to know which children can be expected to have abdominal pain in the near future. Knowledge of the duration of pain before consultation might be a predictor of poor prognosis. Furthermore, we feel that children who continue to have CAP 1 year after the initial complaint need follow-up care or treatment irrespective of the duration of CAP beyond 1 year.

In the Dutch health care system, all inhabitants are registered in a general practice, and specialist care in secondary or tertiary settings requires referral by a primary care physician. As a result, our cohort is likely to represent exclusively nonreferred children. Our study shows that most children complaining of abdominal pain to their physician did not have organic disease; therefore, the results are generalizable to children who have abdominal pain of functional origin. Children were not eligible to participate if they had consulted their primary care physician in the previous 3 months. Our study findings therefore relate to children with a new episode of abdominal pain. 
A postal survey in the UK investigating chronic pain in children showed that $75 \%$ of the respondents (clinicians and general practitioners interested in chronic pain management) opined that most children with chronic pain had a fair to good prognosis. ${ }^{24}$ Our study showed that of children complaining of abdominal pain in general practice, $78.7 \%$ had (or developed) CAP, that a considerable number fulfilled the criteria of CAP 1 year after the initial visit, and one-half of these children were still impaired in their daily functioning. In our opinion this prognosis is not good, and follow-up of these children is warranted.

To read or post commentaries in response to this article, see it online at http://www.annfammed.org/content/11/3/238.

Key words: chronic abdominal pain; primary care, child; prognosis; epidemiology

Submitted May 10, 2012; submitted, revised, July 31, 2012; accepted August 29, 2012.

Funding support: The study was funded by The Netherlands Organisation for Health Research and Development (ZonMW), grant no. 4200.0008

Disclaimer: ZonMW did not have any influence on data collection, analysis, or preparation of the manuscript.

Previous presentations: 2009 NAPCRG Annual Conference, November 14-18, Montreal, Quebec, Canada, and the Wonca Europe 18th World Conference, October 6-9, 2010, Malaga, Spain.

Acknowledgments: The authors thank Metthilde Bos, Toke Mulder, Marlies Luiten, Ellen Duijster and Wilma Bergen Henegouwen for their assistance in data collection and data management.

\section{References}

1. BEACH Program, AIHW General Practice Statistics and Classification Unit. Presentations of abdominal pain in Australian general practice. Aust Fam Physician. 2004;33(12):968-969

2. Gieteling MJ, Lisman-van Leeuwen Y, van der Wouden JC, Schellevis FG, Berger MY. Childhood nonspecific abdominal pain in family practice: incidence, associated factors, and management. Ann Fam Med. 2011;9(4):337-343

3. Chitkara DK, Rawat DJ, Talley NJ. The epidemiology of childhood recurrent abdominal pain in Western countries: a systematic review. Am J Gastroenterol. 2005;100(8):1868-1875.

4. Apley J, Naish N. Recurrent abdominal pains: a field survey of 1,000 school children. Arch Dis Child. 1958;33(168):165-170.

5. Størdal K, Nygaard EA, Bentsen BS. Organic abnormalities in recurrent abdominal pain in children. Acta Paediatr. 2001;90(6):638-642.

6. Hyams JS, Burke G, Davis PM, Rzepski B, Andrulonis PA. Abdominal pain and irritable bowel syndrome in adolescents: a communitybased study. J Pediatr. 1996;129(2):220-226.
7. Petersen S, Hägglöf BL, Bergström El. Impaired health-related quality of life in children with recurrent pain. Pediatrics. 2009;124(4): e759-e767.

8. von Baeyer CL, Walker LS. Children with recurrent abdominal pain: issues in the selection and description of research participants. J Dev Behav Pediatr. 1999;20(5):307-313.

9. Rasquin A, Di Lorenzo C, Forbes D, et al. Childhood functional gastrointestinal disorders: child/adolescent. Gastroenterology. 2006; 130(5):1527-1537.

10. Gieteling MJ, Bierma-Zeinstra SM, Passchier J, Berger MY. Prognosis of chronic or recurrent abdominal pain in children. J Pediatr Gastroenterol Nutr. 2008;47(3):316-326.

11. Huertas-Ceballos AA, Logan S, Bennett C, Macarthur C. Dietary interventions for recurrent abdominal pain (RAP) and irritable bowel syndrome (IBS) in childhood. Cochrane Database Syst Rev. 2009;(1)(Issue 1):CD003019.

12. Huertas-Ceballos A, Logan S, Bennett C, Macarthur C. Pharmacological interventions for recurrent abdominal pain (RAP) and irritable bowel syndrome (IBS) in childhood. Cochrane Database Syst Rev. 2008;(1)(Issue 1):CD003017.

13. Huertas-Ceballos A, Logan S, Bennett C, Macarthur C. Psychosocial interventions for recurrent abdominal pain (RAP) and irritable bowel syndrome (IBS) in childhood. Cochrane Database Syst Rev. 2008;(1)(Issue 1):CD003014.

14. Spee LAA, van den Hurk AP, van Leeuwen $Y$, et al. Childhood abdominal pain in primary care: design and patient selection of the HONEUR abdominal pain cohort. BMC Fam Pract. 2010;11:27.

15. Whaley L, Wong D. Nursing Care of Infants and Children. 3rd ed. St. Louis, MO: CV Mosby Comp, 1987:1070.

16. Firestone M, Moya J, Cohen-Hubal E, Zartarian V, Xue J. Identifying childhood age groups for exposure assessments and monitoring. Risk Anal. 2007;27(3):701-714.

17. Huang RC, Palmer LJ, Forbes DA. Prevalence and pattern of childhood abdominal pain in an Australian general practice. J Paediatr Child Health. 2000;36(4):349-353.

18. Howell S, Poulton R, Talley NJ. The natural history of childhood abdominal pain and its association with adult irritable bowel syndrome: birth-cohort study. Am J Gastroenterol. 2005;100(9):2071-2078

19. Grøholt EK, Stigum H, Nordhagen R, Köhler L. Recurrent pain in children, socio-economic factors and accumulation in families. Eur J Epidemiol. 2003;18(10):965-975.

20. Hotopf M, Carr S, Mayou R, Wadsworth M, Wessely S. Why do children have chronic abdominal pain, and what happens to them when they grow up? Population based cohort study. BMJ. 1998;316(7139):1196-1200.

21. Petersen S, Bergström E, Brulin C. High prevalence of tiredness and pain in young schoolchildren. Scand J Public Health. 2003;31(5): 367-374.

22. Alfvén $\mathrm{G}$. The covariation of common psychosomatic symptoms among children from socio-economically differing residential areas. An epidemiological study. Acta Paediatr. 1993;82(5):484-487.

23. Perquin CW, Hazebroek-Kampschreur AA, Hunfeld JA, et al. Pain in children and adolescents: a common experience. Pain. 2000;87(1): $51-58$.

24. Bhatia A, Brennan L, Abrahams M, Gilder F. Chronic pain in chil dren in the UK: a survey of pain clinicians and general practitioners. Paediatr Anaesth. 2008;18(10):957-966. 